\title{
Pertussis Toxin
}

National Cancer Institute

\section{Source}

National Cancer Institute. Pertussis Toxin. NCI Thesaurus. Code C736.

A class of biologically active protein toxins expressed by Bordetella pertussis, which causes the symptoms of whooping cough. Following bacterial expression or administration of pertussis toxin (PT), the B component of the toxin binds to a cell surface receptor. The entire toxin is internalized by the cell, it is transported to the endosome and then undergoes retrograde transport to the endoplasmic reticulum (ER). During transport the A component undergoes a conformational change that activates its enzymatic activity. The active A component catalyzes the ADP-ribosylation of Gi alpha subunits of heterotrimeric G proteins, which inhibits $\mathrm{G}$ protein-coupled receptor signaling and adenylate cyclase activity. This leads to increased concentrations of cyclic AMP (cAMP), which dysregulates insulin production, chemokine synthesis, and the innate immune response. PT may have various therapeutic effects, including antihypertensive, antiviral, and autoimmune inhibition, but the mechanism(s) of action by which PT exerts its therapeutic activities has not yet been fully elucidated. 